\title{
Metabolic changes in colorectal carcinomas are key factors for the early detection of neoplastic change
}

\author{
Voke Peter Orugbo, ${ }^{1}$ Esaba Emmanuel Akpo ${ }^{2}$ \\ ${ }^{1}$ Department of Chemical Pathology; ${ }^{2}$ Department of Surgery, Delta State University, Abraka, Delta State, \\ Nigeria
}

\begin{abstract}
Potential metabolic biomarkers have been developed by the use of modern analytical techniques and nanotechnology in metabolomics, providing insight into the pathophysiological basis and changes, tumorigenesis, and molecular mechanisms that underpin better therapeutic, monitoring, and prognostic evaluations of colon malignancies. This would allow early detection and characterization of malignant colon tumors and could reduce the risk of mortality and morbidity of colorectal carcinomas. Based on their association with certain metabolic pathways linked to malignancies, a number of tumor markers have been designed. Whereas some have been associated with only one cancer type, while others are associated with many different forms of cancer. No tumor marker has been found to have universal application as a metabolism-related marker; although some are circulating tumor markers found in blood, urine, stool, or other body fluids, others may be found in the specific tumors themselves. This paper
\end{abstract}

Correspondence: Voke Peter Orugbo, Department of Chemical Pathology, Delta State University, Abraka, Delta State, Nigeria.

E-mail: drorugbopeter@gmail.com

Key words: Metabolomics; colorectal carcinomas; metabolites.

Acknowledgements: The authors are grateful to Miss LovethErhijodo, research assistant, for type-setting themanuscript.

Conflict of interest: The authors declare no conflicts of interest

Availability of data and materials: All data generated or analyzed during this study are included in this published article.

Ethics approval and consent to participate: Not applicable.

Informed consent: Written informed consent was obtained from a legally authorized representative(s) for anonymized patient information to be published in this article.

Received for publication: 2 February 2021.

Revision received: 14 May 2021

Accepted for publication: 14 May 2021.

This work is licensed under a Creative Commons Attribution NonCommercial 4.0 License (CC BY-NC 4.0).

(C) Copyright: the Author(s), 2021

Licensee PAGEPress, Italy

Annals of Clinical and Biomedical Research 2021; 2:134

doi:10.4081/acbr.2021.134 addresses a number of associated metabolic changes linked to colorectal cancers and potential applications for disease condition diagnosis, monitoring, treatment, and prognosis.

\section{Introduction}

Colorectal Cancer (CRC) is one of the most common cancers worldwide, with more than one million new cases diagnosed each year. Carcinoma of the colon and rectum is the second most common cancer in the United States, with lung cancer being the most common. It is estimated that 130,200 new cases of colorectal cancer will be examined each year, with 56,300 people dying from the disease. CRCis no longer a Western disorder today. Over the past six decades, it was previously considered a rarity in black African; it has dramatically increased incidence among indigenous Africans. ${ }^{1} \mathrm{CRC}$ in countries with a previously established low incidence is a growing public health issue, with rising rates. The actual incidence of colorectal cancer and prevalence data from Africa are believed to underestimate the burden of the disease. This underestimation is most likely correlated with methods of data collection, lack of centralized processes, limited healthcare provider awareness, and patient-related factors such as late presentation. The incidence of CRC is growing globally, while its ranking has remained the same between 2008 and 2012 across all forms of cancer.

In Africa, although slight regional variation in prevalence has been noted, CRC is the fourth most common fatal malignancy. Most cases are due to inadequate dietary habits, host immunity, and causes such as smoking, low levels of physical activity, and obesity in the lifestyle. As risk factors for the onset of cancer, other gastrointestinal disorders, such as inflammatory bowel disease characterized by chronic inflammation, mucosal disturbance and excessive production of reactive oxygen species, function.

Much of the energy is provided by the electron transport chain stage (32-34 ATP molecules, compared to only 2 ATP for glycolysis and 2 ATP for the cancer cycle). However, cancer cells differently prioritize energy sources to facilitate their rapid growth and proliferation. This is even more predicated when the cancer cells proliferate.

Proliferation is a major part of the creation and growth of cancer. This is manifested by the altered expression and/or behavior of proteins associated with the cell cycle. A fibrogenic response and the development of a hypoxic environment that favors the survival and proliferation of cancer stem cells are correlated with early steps in tumor development. Alterations in cell metabolism may be part of the survival strategy of cancer stem cells. The cancer cell embodies features that allow it to survive and proliferate abnormally beyond its natural life span.

The development of cancer results from the selection of mutation(s) cells which provide survival and proliferative benefits. In response to ever-changing hostile microenvironments, the hypoxia-inducible factors, $H I F-1$ and $H I F-2$, are upregulated, where 
reduced oxygen tension, reduced glucose concentrations and low $\mathrm{pH}$ are found. Trans HIFs activate genes that promote tumor growth by, among other functions, mediate intermediate metabolism (glycolysis) and regulate $\mathrm{pH} .{ }^{2,3}$

The renewed attention given to metabolic processes related to malignant cells has steered a drive towards an understanding of metabolic phenotyping in the histochemical pathology of cancer tissues. ${ }^{1}$ Neoplastic cells go through a series of metabolic transformations to maintain rapid cell division, multiplication, and propagation. ${ }^{2}$ The aftermath of this metamorphosis is that several and varied metabolic phenotypes emanate in the cancer cells which are quite dissimilar when compared with non-neoplastic cells. ${ }^{3} \mathrm{~A}$ comprehensive understanding of the natural science of tumour growth would be a mirage without identifying the gamut of metabolites and their diverse nature. This information is vital and more so that it could also enable scientists to advance techniques for cancer care while using these metabolites as diagnostic and prognostic markers. ${ }^{1,4}$

Colorectal carcinomas are malignant tumors of the colon. Their growth involves production or increased production of some metabolites and/or dysregulation/decreased production of some other metabolites. ${ }^{5}$ These metabolites cause metabolic changes through effects in general or specific metabolic processes occurring in the body. ${ }^{6}$ The change in metabolic processes often produces deleterious effects. Estimation of the levels of the metabolites could serve as prognostic markers in colorectal carcinoma. ${ }^{2,7}$

Colorectal cancer is a frequently occurring cancer in the general population worldwide among which the sporadic subtype forms $70 \%{ }^{8}$ As it were, colorectal carcinoma emerges gradually via a persistent build-up of numerous mutations at varying points occurring in oncogenes, tumorsuppressor genes, and intermediary metabolic pathways, including normal metabolic pathways. ${ }^{8}$ Colon cells are designated with a definite outline in metabolic processes that confer on them characteristics that distinguish malignant from non-malignant tissues and delineate cancer types into clinicopathologic physiognomies and thus, response to colorectal cancer-related interventions. ${ }^{1,9}$

There is the need for newer non-invasive methods focused on early detection of colorectal cancer particularly in the face of possible complications that may follow colonoscopy, which in combination with histopathology forms the current gold standard in the diagnosis of colorectal carcinoma. , $^{4,11}$

\section{Metabolic chemicals in colorectal cancer}

Metabolic changes in colorectal carcinomas produce deleterious effects through increased generation of metabolites outlined above which cause inflammation, oxidative stress, angiogenesis, and act as acute positive reactants. These features promote tumor growth and proliferation. ${ }^{12-14}$

It has been reported that a continuum of metabolic eccentricities associated with an initial remediable CRC stage is pertinent for a successful and timely application of molecular diagnostic and therapeutic methodologies whose ultimate goal is to enhance quick recovery and advance the chances of survival. ${ }^{4,12}$

Some of these metabolites include: Hypotaurine $\beta$ - Alanine, Glutamate, Kynurenine, Cysteine, 2-Aminobutyrate, Palmitoleate, 5-Oxoproline, Aspartate, Hypoxanthine, Lactate, Myristate, Glycerol, Uracil, Putrescine, Myo-inositol, Spermidine, Homocysteine, 4-Aminobutyrate, Asparagine, Glycerate, Nicotinamide, Adenosine monophosphate (AMP), Ascorbic acid, Glucose, Xylose, Glycine, Glyceraldehyde, Ornithine,
Phosphate, Laurate, Galactose, 3-Methyl-3-hydroxybutyrate, Methiotinamide and 2-Aminoadipate. ${ }^{5,13}$

Metabolites that are reduced in concentrations are usually negative acute phase reactants and are anti-inflammatory and antioxidative. These get reduced through fighting inflammatory processes and clearing debris effects like necrosis. ${ }^{15}$

From aerobic respiratory processes to anaerobic processes in energy production, the metabolic changes that occur early in colorectal carcinomas tend to produce the Warburg effect. Warburg effect is a survival mechanism in hypoxic situations where energy generation in highly proliferative cells shift from normal colon tissues to malignant ones. ${ }^{6,16}$ Glycolysis is enhanced but with shunting of pyruvic acid to lactic acid production instead of glyceraldehyde-3-phosphate and dihydroxy-acetone phosphate which finally produce more metabolic in Adenosine Triphosphate (ATP). This will cause metabolic acidosis from lactic acid. Metabolic acidosis milieu stimulates more cancerous cell proliferation, producing a vicious cycle causing inflammation, necrosis, and cell death. ${ }^{17}$ Overall, a lot of increased metabolites (pro-oxidants) and decreased metabolites (anti-oxidants) cause deleterious effects. There is increased shunting of glycolytic intermediates from entering the tricarboxylic acid cycle to other metabolic pathways that yield products used in Deoxyribonucleic Acid (DNA), Ribonucleic Acid (RNA), and lipids (especially fatty acids) synthesis to meet the needs of increased cellular proliferation.,18 Glucose is a reduced metabolite, and with shunting of glycolytic products to the Cori cycle because of the Warburg effect, there is increased fatty acids use to generate energy. ${ }^{7-9,19}$

Cancer cells develop characteristic metabolic pathways yielding processes like angiogenesis with intense proliferation and fewer energy needs. As demonstrated in the Warburg effect, when aerobic glycolysis is augmented or amplified several events unfold including distinguishing manifestations, transmutations, and posttranslational modification of enzymes catalyzing various metabolic processes in the body. ${ }^{10,21}$ These events are due to survival processes in the highly proliferative cancer cells resulting in cancer growth and metastases. There is also affectation of proliferationpromoting oncogenes with altered metabolic changes including significantly higher levels of lactate in colorectal cancer samples compared with non-cancer cells. 4,9,10,22,23

The Kynurenine Pathway produces tryptophan catalyzed by Indoleamine 2,3-Dioxygenase (IDO). This enzyme mediates tumor-related immune tolerance, involved in preventing tumor cells from immune attack. ${ }^{14}$ A substantial amplification in the expression of the IDO gene is seen in cancerous colon tissues, in contrast, to healthy colon tissues. High IDO expression results in the release of higher levels of kynurenine. Nicotinamide adenosine dinucleotide is produced from tryptophan in the kynurenine pathway. A hyper-functional kynurenine metabolism also produces more for nicotinamide adenosine dinucleotide the electron transport chain to cope with high energy demands for the fast turnover of proliferative cancer cells. ${ }^{15}$

These distinct metabolic signature metabolite markers are applicable in predicting response to interventions in colorectal carcinoma patients. There are metabolic aberrations with genetic signature shown to have occurred through mutations leading to a sustained and excessive cell multiplication in colorectal carcinoma. These metabolic adaptations show features of the Warburg effect and other survival metabolic processes like angiogenesis and evasion of immune attack that promote tumor cells proliferation and metastases even in a hostile metabolic milieu that would have stimulated cellular death through apoptosis and necrosis. ${ }^{11,12,26} \mathrm{It}$ has also been reported that colonic cancerous tumor cells have 
high amounts of lactate and other metabolites expressed by malignant tissues like aspartate and taurineare richer in taurine. ${ }^{13,27}$

Increased metabolic activities in tumor cells produce Reactive Oxygen Species (ROS). These are usually cellular metabolic products. Greater levels of ROS occur in malignant tumors. Malignant tumor tissues get transformed metabolically, and this makes them adapt to the varied metabolic milieu, which may often occur anaerobically after glycolysis. ${ }^{14,28}$ There are increased glutathione precursors like cysteine and glycine. ${ }^{15,28,29}$

\section{Application of oxidative stress biomarkers}

Oxidative stress biomarkers like ophthalmate reflect GSH depletion through gamma-glutamylcysteine synthase activation. Gamm-glutamyl cysteine synthase along with glutathione synthetase catalyzes ophthalmate production, glutamate, glycine, and 2-aminobutyric acid. Glutathione synthetase and 2-aminobutyric acid are markedly raised in malignant colon tissues, which suggests increased ophthalmate synthase activities in colorectal cancer tissues. ${ }^{16,17,30}$ Also increased is 2-aminobutyric acid in epithelial ovarian carcinoma tissues compared with normal tissues of the ovary. Other enzymes elevated in malignant colon tumors include glutathione $\mathrm{S}$ transferase pi 1 (GSTP1), GSH peroxidase 1 (GPX1), GSH reductase (GSR), and gamma-glutamylcyclotransferse (GGCT) compared with non-tumor controls. Aminopeptidase $\mathrm{N}$ (ANPEP) is an enzyme that is reduced in glutathione metabolism. ANPEP facilitates the breakdown of cysteinyl glycine to cysteine and glycine, and thus, cysteinyl glycine levels are lower in colorectal cancer tissues when compared with those of the control tissues. ${ }^{18,19,31}$ The GSH redox cycle is also coupled with the NADP/NADPH transformation, which is needed in enhanced synthesis of fatty acids. An increased NADPH synthesis through the pentose phosphate pathway with increased levels of 5-oxoproline, and 2-aminobutyric acid are also seen in malignant tumors of the colon. Increased oxidative stress is usually not only associated with increased production of fatty acids but also the elevation of metabolites involved in anti-oxidation processes. ${ }^{32}$ Increased fatty acid production usually leads to the accumulation of 3-hydroxybutyrate supporting the hypothesis of enhanced metabolic changes in tumor cells. ${ }^{10,33,34}$

Metabolic alterations that occur during tumorigenesis in colorectal cells show an elevation in the amount and types of protein precursors (usually amino acids) and fats in malignant tissues, indicating that there is a higher energy requirement during high proliferation seen in these tissues. Glucose and inositol are reduced in neoplastic proliferation. ${ }^{13}$ Besides, the accumulation of hypoxanthine and xanthine, and the decreased uric acid are features showing substitution of the purine metabolic pathway by the salvage pathway in CRC. ${ }^{13}$ It is also found that lipid biomolecules, phosphates, and several amino acids levels are elevated. On the contrary, glucose, uric acid, and inositol levels are markedly reduced. Glutamate and proline are among the elevated amino acids that favor the hypothesis that increased proliferative growth of malignant tumor cells is facilitated by increased protein synthesis. ${ }^{14,37}$ There is increased transport of glutamine into cells for glutamate production through glutaminase. This occurs at a faster rate when compared to non-cancerous colorectal cells showing that an increased rate of protein synthesis is vital to the survival of malignant colorectal cells in these rapidly growing tumor cells. Glutaminase is increased and this leads to elevation of glutamate levels in malignant colonic tumor cells. ${ }^{15,38}$

An oxidoreductase is involved in purine catabolism that pro- duces xanthine from hypoxanthine with subsequent uric acid production. This enzyme is well expressed in gastrointestinal epithelial cells. Nonetheless, this is decreased in gastrointestinal epithelial cells of malignant colorectal tumors. ${ }^{39}$ This is strongly correlated in the degree of expression in the size of the tumor, level of tumor involvement, advanced stage, and risk of metastasis. This observation is not unconnected with the fact that the salvage pathway of purine nucleotides synthesis uses less ATP (i.e. less energy use) compared to de novo purine nucleotides biosynthesis metabolic change in which colorectal neoplastic cells effectively shunt purine bases to the salvage pathway and confers higher proliferative and survival rate over non-malignant colorectal epithelial cells. ${ }^{16,40}$

Choline and phosphocholine levels are increased in malignant colorectal tumors. The increase is associated with new cell membrane synthesis to meet the need for membrane lipids due to accelerated cellular proliferation. Increased choline and phosphocholine synthesis are also correlated with increased inflammatory processes. ${ }^{17,41}$

\section{Fatty acid synthesis and fatty acid synthase as a target for tumor control}

Fatty acid synthesis through generating metabolic processes produce building blocks used in cellular membranes synthesis required to meet fast growth turnover of malignant colorectal tumor cells which are also mainly used in membrane lipid composition, like choline and phosphocholine. ${ }^{18,42}$ The enzyme, Fatty Acid Synthase (FAS), involved in lipid synthesis, is highly expressed in malignant colon tumors and it has been observed that inhibition of FAS is associated with decreased angiogenesis in malignant colon tumors and metastatic processes to other organs like the lungs and liver indicating that inhibiting FAS may be a therapy target in the management of cancers of the colon. ${ }^{43}$

The field of metabolomics is showing differences between non-malignant and malignant tissues through pathophysiological knowledge of tumor growth. ${ }^{19,44}$ This involves metabolomics profiling and changes seen in healthy control persons correlated with diseased cases/patients. Some metabolic profiling and changes are influenced by environmental factors and biological alterations including diet, lifestyle, medications, and chronic diseases. ${ }^{45}$ The use of modern analytical techniques and nanotechnology in metabolomics has produced potential metabolomics biomarkers, giving insight into the pathophysiological basis and changes, tumorigenesis, and molecular mechanisms that underpin better therapeutic, monitoring, and prognostic evaluations of malignant tumors of the colon. ${ }^{46}$ Thus, enabling early detections and characterization of malignant colon tumors to reduce mortality and mor-

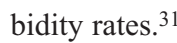

Leucine, isoleucine, and glutathione are elevated in all stages of colorectal carcinomas. Neoplastic transformations and proliferation of colon tissues are affected by biological, chemical, and environmental exposure changes, more studies are needed in this field. ${ }^{34,47}$

Distinct metabolic markers are increasingly being evaluated for colorectal carcinomas and used in diagnosis, chemotherapy, monitoring, and prognosis in the management of colorectal carcinoma. ${ }^{11,35}$ There are metabolic aberrations at the points where genes are expressed that indicate alterations in the metabolism of colorectal cancer cells showing features beyond the Warburg effect 
that addresses only the amplified energy demand via an ideal glycolytic process, in providing support to the increased needs of energy, macromolecular precursors, and in redox balance homeostasis in the presence of intense oxidative stress. ${ }^{12,36}$

\section{Early markers for colorectal carcinoma}

Tumor markers, also known as biomarkers, are chemical compounds produced by cancer cells or other cells in the body when they are exposed to cancer. Colorectal cancer tumor markers can be found in the blood. A blood test is used to determine whether they are present in the tumor tissue or not. They are usually cancer-related proteins that can be clinically useful in cancer patients. A high tumor marker level indicates that cancer may be present in the body, but a high tumor marker level alone does not indicate a diagnosis.Several colorectal cancer markers have been identified and are accepted in standard clinical practice, namely tissue polypeptide specific antigen (TPS), tumor-associated glycoprotein-72 (TAG-72) and hematopoietic growth factors (HGFs) ${ }^{48}$ Examples of markers found in the blood are carcinoembryonic antigen (CEA), carbohydrate antigen (CA 19.90), Chromosome $18 \mathrm{q}$ loss of heterozygosity(18qLOH). However,MSI (microsatellite instability), K-RAS and BRAF mutations are examples of Tumor Markers Found in Tumor Tissue.

\section{Tumor markers found in the blood}

\section{Carcinoembryonic antigen (CEA) levels}

CarcinoembryonicAntigen (CEA) is a protein usually present in the blood of adults at very low levels. A glycoprotein oncofetal antigen is a Carcinoembryonic Antigen (CEA) that is expressed in many epithelial tumors. This relatively cheap blood testwas part of the most recommendedsurveillance strategies. ${ }^{49}$ CEA is a glycoprotein which is produced in the big bowel cells. During diagnosis, seventy percent of CRC patients have elevated levels of CEA, which makes it a very good predictor for disease management and monitoring after resection. In some kinds of cancer and non-cancerous (benign) conditions, the CEA blood level may be increased. A CEA test is most commonly used for colorectal cancer. CEA is not something that a doctor orders on a daily basis. It is completed if the doctor suspects cancer or if the aim is to determine if cancer treatment is effective. In other cases, a CEA test is recommended by the doctor to determine whether a recurrence of cancer has occurred.

While CEA is generally considered a marker of cancer, in a number of benign conditions, including hepatitis, pancreatitis, obstructive pulmonary disease and inflammatory bowel disease, its concentrations may also be elevated. According to generally accepted units of measurement, blood antigen levels of up to 5 $\mathrm{ng} / \mathrm{mL}$ are considered natural. These values have been shown to be increased by up to $10 \mathrm{ng} / \mathrm{ml}$ in smokers in cases of ulcerativecolitis or liver cirrhosis. ${ }^{50}$ In colorectal cancer, this tumor marker is the most widely used. It can be tested to predict prognosis prior to surgery, used during therapy to determine response to treatment, or to monitor for recurrence after completion of therapy.

The use of CEA is not always one hundred percent correct. This is because a number of reports have shown impact of metabolic factors including obesity, insulin resistance, glucose.intolerance, hypertension, and dyslipidemia, and is associated with high morbidity and mortality. ${ }^{51,52} \mathrm{As}$ a causal risk factor for cardiovascular disease and several types of cancer, such as breast, pancreatic and colon cancers, metabolic syndrome has also been reported..$^{53,54}$

One recent study indicated that obesity was associated with lower levels of CEA, ${ }^{55}$ which in the CEA test may result in loss of sensitivity and diagnostic accuracy. Therefore, due in part to their association with obesity, interrelationships between CEA levels and the individual component of metabolic syndrome may exist. The relationship between an individual and the combined components of metabolic syndrome and the degree of CEA is currently unknown.

Depending on the performing laboratory, the lower limit of normal varies but usually ranges from 2.5 to $5 \mathrm{ng} / \mathrm{mL}$. In more than $87 \%$ and $<5 \mathrm{ng} / \mathrm{mL}$, serum CEA was $<2.5 \mathrm{ng} / \mathrm{mL}$ in more than 95 percent of 1,020 subjects attending primary prevention clinics. ${ }^{56} \mathrm{In}$ smokers and patients with inflammatory conditions, elevated CEA levels are more normal, but rarely exceed $10 \mathrm{ng} / \mathrm{mL} .{ }^{57,58}$ In a number of other carcinomas, including lung, breast, gastrointestinal and gynecologic cancers, the test may also be elevated. ${ }^{59}$

CEA's sensitivity and specificity depends on the type immunoassay used.the patient population studied, and the performing facility. ${ }^{60}$ In early colon cancer patients, the sensitivity of CEA is low and increases with the growing stage of the disease.Fletcher ${ }^{61}$ recorded that sensitivity was 36 percent with a specificity of 87 percent for a CEA $>2.5 \mathrm{ng} / \mathrm{mL}$ in patients with stage I and II disease, after reviewing the sensitivity and specificity of the CEA test at different stages of the disease. At similar CEA stages, the sensitivity for stage III and IV diseases was 74 percent and 83 percent, respectively. ${ }^{61}$

The specificity of elevated CEA cut-points increased while the responsiveness decreased ( $5 \mathrm{ng}$ and $10 \mathrm{ng}$ ). This test is unsuitable for primary colorectal cancer screening due to its low reliability in early colorectal cancer and its imperfect specificity in the general population. It is normal to expect a higher risk of relapse in patients with an elevated preoperative CEA level, given the positive correlation between elevated CEA and the stage of colorectal cancer at initial presentation. This then makes a good prognostic marker for CEA.

\section{Carbohydrate Antigen (CA19-9)}

Carbohydrate Antigen (CA19-9) is a cancer antigen that often detects elevated serum levels in the case of colorectal cancer. This test tests the volume in the blood of a protein named CA 19-9 (cancer 19-9 antigen). CA19-9 is a type of marker for tumors. In response to cancer in the body, tumor markers are substances made by cancer cells or by normal cells. In the blood of healthy people CA 19-9 levels are low. High levels of CA 19-9 are often a sign of pancreatic cancer. But occasionally, other forms of cancer or some noncancerous conditions, including cirrhosis and gallstones, can be indicated by elevated levels.

It is a tumor marker that is observed for metastatic colon cancer at elevated serum concentrations. In clinical practice, CEA and CA19-9 are used, but we have to acknowledge the fact that they are not unique to early colon cancer detection, which means that they cannot be used in the diagnosis of in situ carcinoma. ${ }^{62}$

The serum level of CA19-9 in these patients is poorly understood, raising concerns about colorectal carcinoma progression and relapse. However, some studies have found serum CA19-9 measurement to be useful, ${ }^{63-65}$ while others have found it to be ineffective, ${ }^{66-68}$ and CA19-9 measurement is not recommended. Kawamura et al. ${ }^{69}$ concluded that the measurement of serum 
CA19-9 should be omitted regardless of clinical setting if the result of the first measurement of serum CA19-9 was less than the lower limit. Even though CA19-9 synthesis has increased in a certain group of patients with colorectal carcinoma, particularly those with advanced disease, Kawamura et al. ${ }^{69}$ showed that serum CA19-9 leveled remained below the measurable limit, regardless of the stage of diagnosis, progression and recurrence. Whereas CA 19-9 is a tumor marker expressed in colorectal cancers, it has also been shown to be of significant prognostic value, especially in advanced cases.

\section{Chromosome 18q loss of heterozygosity $(18 q \mathrm{LOH})$}

This is often applied in patients with stage II or III colorectal cancer; can influences prognosis. CRC is the result of the progressive accumulation of multiple genetic and epigenetic aberrations within the cells. CRC is a chronic group and the genetic makeup of the malignancies ascertain their prognosis and response to targeted therapies. Relatively early diagnosis and appropriate therapeutic strategies based on current understanding of the molecular characteristics of CRC are important for the proper treatment of CRC.

Like several other solid tumors, CRC is a heterogeneous disorder in which multiple subtypes can be differentiated by their particular clinical and/or molecular characteristics. Most sporadic CRCs ( $>85 \%$ ) have chromosomal instability (CIN), with variations in the number and structure of chromosomes. ${ }^{70,71}$ These modifications include chromosomal segment gains or losses, chromosomal rearrangements and heterozygosity loss $(\mathrm{LOH})$, resulting in variations in the number of gene copies (CNVs). ${ }^{69-71}$

Colorectal cancer is the second most common cancer in a number of Western countries. Outcome among these patients is often poor, with an average 5-year relative survival rate of around $50 \% .^{72}$

In the late carcinogenic phase, allelic loss at chromosome $18 \mathrm{q}$ is observed in approximately 70 percent of primary $\mathrm{CRC}^{73-78}$ and is regarded as a weak prognosis marker for viability in CRC patients. ${ }^{79}$ In CRC, Loss Of Heterozygosity (LOH) on chromosome $18 \mathrm{q}$ is common and has also been linked to an increased risk of poor prognosis in stage II tumors.

Allelic loss (Loss Of Heterozygosity, LOH) in the long arm of chromosome 18 can be detected in approximately 60-70 percent of cases of colorectal cancer. The most frequent area of loss is where the candidate tumor suppressor genes MADR2, DPC4, and DCC are known to reside. ${ }^{80,81}$ The area has not been fully examined and it is believed that other tumor suppressor genes are likely to be found there in the near future. ${ }^{82}$

The existence of candidate tumor suppressor genes whose inactivation may play a significant role in CRC is indicated by the high frequency of allelic deletions involving chromosome $18 \mathrm{q} .{ }^{80-}$ 83 DCC, which encodes a portion of the neutrin-1 receptor in the $18 \mathrm{q} 21.2$ chromosome band, was proposed as a putative tumor-suppressor gene. ${ }^{80}$

Chang et al. ${ }^{84}$ examined the prognostic importance and frequency of $\mathrm{LOH}$ near regions containing essential genes in the development of colorectal cancer at 14 genetic locations. In 78.8 percent of the tumors, LOH occurred in one such genetic locus. Tumor suppressor gene locus [TP53.alu (65\%), DCC (64.3\%), D8S254 (51.7\%) and APC (47.8\%)] were determined as regions observed at the highest frequency of $\mathrm{LOH}^{84}$ It has also been found that high-frequency LOH correlates with the high metastatic risk of colorectal cancers. The association between $\mathrm{LOH}$ in regions where APC and DCC genes are located and micro metastasis in per colonic lymph nodes has also been studied in another study ${ }^{83}$ and no correlation has been found.

\section{Tumor markers found in tumor tissue}

\section{Microsatellite Instability (MSI)}

According to Iacopetta et al. ${ }^{85}$ approximately 20 percent of right-sided colon cancers and 5 percent of left-sided colon and rectal cancers have a deficient DNA mismatch repair system. This results in the widespread accumulation of mutations to nucleotide repeats, some of which occur within the coding regions of cancerrelated genes such as TGF $\beta$ RII and BAX. A standardized definition for Microsatellite Instability (MSI) based on the presence of deletions to mononucleotide repeats is gaining widespread acceptance in both research and the clinic. ${ }^{85} \mathrm{CRC}$ with MSI characterized histologically by an abundance of tumor-infiltrating lymphocytes, poor differentiation and a signet ring or mucinous phenotype.

Tiny tandem repetitive DNA sequences found in the genome are microsatellites. MIN is caused by the failure of the DNA mismatch repair system. This can result in microsatellite growth, shrinkage, deletion, and random insertion. ${ }^{86}$

In both cancer initiation and progression, genomic and MSI play critical roles. This instability can be genetically expressed at many different stages, ranging from basic sequence changes of Deoxyribonucleic Acid (DNA) to structural and numerical anomalies at the chromosomal level. Although it is a well-established molecular marker for Lynch syndrome patients, MSI has only been slowly recognized as a clinically relevant feature of tumor biology. ${ }^{87}$

One of the common properties of colorectal cancer is genetic instability. In colorectal cancer, three major types of genetic instability were identified. ${ }^{80,88}$ Due to differences in DNA mismatch repair genes, microsatellite instability occurs, while Chromosomal Instability (CIN) is characterized by significant chromosomal changes arising in cell division and typically includes mutations of $\beta$-catenin and Adenomatous Polyposis Coli protein (APC).

It is well known that tumors with microsatellite instability contain more mutations than other tumors. Tumors of chromosomal instability and microsatellite instability were primarily regarded as similarly special since tumors of microsatellite instability typically have constant and diploid karyotypes. ${ }^{89,90}$ Trautmannet al. ${ }^{91}$ found that about 50 percent of tumors with inherited MSI-Hhave similar chromosomal aberration levels.

Several methods are well known to identify tumors with MSI and are being used as a clinical diagnostic tool. MSI-specific microsatellite repeats are observed by PCR amplification. MSI can also be identified by methods of gene expression analysis. MMR protein immunohistochemical analysis has become a common method for the identification of MSI in diagnostic centers and as an alternative to Lynch syndrome genetic testing. ${ }^{92}$ Tumors that may benefit from immunotherapy have been identified in patients with high Microsatellite Instability (MSI-H)/Mismatch Repair Deficiency (dMMR) and MSI can be used as a genetic instability of a tumor detection index.

\section{K-RAS and BRAF mutations}

Clinical trials have shown that not all EGFR overexpression patients respond to the aforementioned monoclonal antibodies. This resistance is partly caused by mutations in oncogenes coding for the downstream proteins. The anti-EGFR monoclonal antibod- 
ies show the strongest response in CRCC when the downstream effectors of EGFR signaling, KRAS, BRAF PI3K, and PTEN, are all unmutated (quadruple negative).

KRAS and BRAF are part of a pathway called MAP kinase (MAPK). Cell proliferation, differentiation, senescence, and apoptosis are regulated via the RAS/RAF/MAPK pathway. HRAS, NRAS, and KRAS are included as RAS oncogenes. In CRC, KRAS is the most often mutated RAS family member and is mutated in $40 \%$ of sporadic CRCs.

Weinberg ${ }^{93}$ postulated that the pathway (or adenoma-carcinoma sequence) of Chromosomal Instability (CIN) follows a predictable progression of genetic and corresponding histologic changes. The genomic changes include proto-oncogene (K-Ras) activation and inactivation of at least three genes for tumor suppression, namely, loss of APC (chromosome region 5q21), loss of p53 (chromosome region 17p13), and loss of heterozygosity in the long arm of chromosome 18 (18q LOH).

KRAS is mutated in about 50 percent of colorectal tumors, and changes in this small GTP-binding protein occur almost uniformly as activating point mutations in codons 12,13 , and, to a lesser degree, 61. Specific mutations in the K-RAS gene will predict whether treatment with many biologic therapies is likely to help a patient or not.

In some clinical trials, KRAS has been explicitly targeted, with negligible activity seen so far. BRAF is a member of the serine/threonine kinase RAF family and mediates cellular responses through the RAS-RAF-MAP kinase pathway to growth signals. Approximately 10 percent of sporadic CRC have recently been found to activate mutations in BRAF and are rare in familial Lynch syndrome CRC. In 4 per cent of MSI-low and 40 per cent of MSIhigh tumors, BRAF mutations were identified. ${ }^{94}$ BRAF mutations are typically associated with a V600E mutation and can predict prognosis after diagnosis of colorectal cancer.

Cancer metabolism refers to the changes in cellular metabolism pathways that are visible in cancer cells compared with most normal tissue cells. Metabolic alterations in cancer cells are numerous and include aerobic glycolysis, reduced oxidative phosphorylation and the increased generation of biosynthetic intermediates needed for cell growth and proliferation.

\section{Conclusions}

Extensive research on tumor markers for cancer diagnosis and control has encouraged increased interest in applying the practice of diagnostic immunopathology in oncology. They are essentially biochemical tumor presence markers that are selectively generated by neoplastic tissue and released into the blood or other body fluids; hence, the role of cellular metabolic changes is imperative in the production of a tumor marker.

For the early diagnosis of colorectal cancer, the identification of tumor markers is valuable; therefore, an ideal tumor marker will be required to have high cancer sensitivity and specificity and satisfy certain requirements. However, the sensitivity of tumor markers to detect early cancer is particularly low, and many tumor markers typically have a high false positive rate of CRC. In addition, single markers are less expensive, but combinations of markers, which may be better alternatives, increase the cost, which is troubling for clinics.

Lastly, provided that metabolic changes in colorectal carcinoma result in metabolite production. Some metabolic profiling and modifications are affected by environmental influences and biological alterations including diet, lifestyle, drugs and chronic diseases. Modifiable risk factors may also become priorities for better risk mitigation.

\section{References}

1. Ferlay J, Shin H-R, Bray F, et al. Estimates of worldwide burden of cancer in 2008: GLOBOCAN 2008. Int J Cancer2010;127;2893-917.

2. DeBerardinis RJ, Chandel NS. Fundamentals of cancer metabolism. Sci Adv 2016;2:1-18.

3. Acker T, Plate KH. A role for hypoxia and hypoxia-inducible transcription factors in tumor physiology. J Mol Med (Berl) 2002;80:562-75.

4. Cheng Y, Xie G, Chen T, et al. Distinct urinary metabolic profile of human colorectal cancer. J Proteome Res 2012;11:135463.

5. Vander Heiden MG, DeBerardinis RJ. Understanding the Intersections between Metabolism and Cancer Biology. Cell 2017; 168:657-69.

6. Fang S, Fang X. Advances in glucose metabolism research in colorectal cancer. Biomed Rep 2016;5:289-95.

7. Granchi C, Bertini S, Macchia M, Minutolo F. Inhibitors of lactate dehydrogenase isoforms and their therapeutic potentials. Curr Med Chem 2010;17:672-97.

8. Yu L, Chen X, Sun X, et al. The Glycolytic Switch in Tumors: How Many Players Are Involved? J Cancer 2017;8:3430-40.

9. Muñoz-Pinedo C, Mjiyad NE, Ricci JE. Cancer metabolism: current perspectives and future directions. Cell Death Dis 2012;3:1-4

10. Boroughs LK, DeBerardinis RJ. Metabolic pathways promoting cancer cell survival and growth. Nat Cell Biol 2015;17:351-9.

11. Almuhaideb A, Papathanasiou N, Bomanji J. 18F-FDG PET/CT imaging in oncology. Ann Saudi Med 2011;31:3-13.

12. Piotto M, Moussallieh F, Dillmann B, et al. Metabolic characterization of primary human colorectal cancers using high resolution magic angle spinning $1 \mathrm{H}$ magnetic resonance spectroscopy. Metabolomics 2009;5:292.

13. Pavlova NN, Thompson CB. The Emerging hallmarks of cancer metabolism. Cell Metab 2016;23:27-47.

14. Armitage EG, Barbas C. Metabolomics in cancer biomarker discovery: current trends and future perspectives. J Pharm Biomed Anal 2014;87:1-11.

15. Serkova NJ, Glunde K. Metabolomics of cancer. Methods MolBiol 2009;520:273-95.

16. Wishart DS, Feunang YD, Marcu A, et al. HMDB 4.0: the human metabolome database for 2018. Nucleic Acids Res 2018;46:D608-D617.

17. Wishart DS, Mandal R, Stanislaus A, Ramirez-Gaona M. Cancer Metabolomics and the Human Metabolome Database. Metabolites 2016;6:23-34.

18. Markley JL, Bruschweiler R, Edison AS, et al. The future of NMR-based metabolomics. CurrOpinBiotechnol 2017;43:3440.

19. Emwas AH. The strengths and weaknesses of NMR spectroscopy and mass spectrometry with particular focus on metabolomics research. Methods MolBiol 2015;1277:161-93.

20. Pakiet A, Kobiela J, Stepnowski P, et al. Lipid: Changes in lipids composition and metabolism in colorectal cancer: a review. Lipids Health Dis 2019;18:29.

21. Stephenson DJ, Hoeferlin LA, Chalfant CE. Lipidomics in translational research and the clinical significance of lipidbased biomarkers. Transl Res 2017;189:13-29.

22. Hamilton SR, Rubio CA, Volgenstein B, et al. Carcinoma of the colon and rectum. In: Hamilton SR, Aaltonen LA, editors. 
World Health Organization classification of Tumours. Pathology and genetics of Tumours of the digestive system. Lyon: IARC Press; 2000: p. 105-120.

23. Strum WB. Colorectal adenomas. N Engl J Med 2016;374:1065-75.

24. Yamagishi H, Kuroda H, Imai Y, Hiraishi H. Molecular pathogenesis of sporadic colorectal cancers. Chin J Cancer 2016;35:4.

25. Grady WM. Genetic testing for high-risk colon cancer patients. Gastroenterol 2003;124:1574-94.

26. Cappell MS. Pathophysiology, clinical presentation, and Management of Colon Cancer. GastroenterolClin N Am 2008;37:1-24.

27. Hornyák L, Dobos N, Koncz G, et al. The role of indoleamine2,3-dioxygenase in cancer development, diagnostics, and therapy. Front Immunol 2018;9:1-8.

28. Zhai L, Spranger S, Binder DC, et al. Molecular pathways: targeting IDO1 and Other tryptophan dioxygenases for cancer immunotherapy. Clin Cancer Res 2015;21:5427-33.

29. Liou G-Y, Storz P. Reactive oxygen species in cancer. Free Radic Res 2010;44:1-31.

30. Liu Y, Hyde AS, Simpson M A, Barycki JJ. Emerging regulatory paradigms in glutathione metabolism. Adv Cancer Res 2014;122:69-101.

31. Iizuka K. The role of carbohydrate response element binding protein in intestinal and hepatic fructose metabolism. Nutrients 2017;9:1-12.

32. Brown RE, Short SP, Williams CS. Colorectal cancer and metabolism. Curr Colorectal Cancer Rep 2018;14:226-41.

33. Araujo SEA, Alves PRA, Habr-Gama A. Role of colonoscopy in colorectal cancer. Rev HospClin 2001;56:23-36.

34. Niederreiter M, Niederreiter L, Schmiderer A, et al. Colorectal cancer screening and prevention - pros and cons. Mag Eur Med Oncol 2019;12:239-43.

35. Wen SH, Yang X. Role of intestinal flora in colorectal cancer from the metabolite perspective: a systematic review. Cancer Manag Res 2018;10:199-206.

36. Vecchia S, Sebastián C. Seminars in cell \& developmental biology metabolic pathways regulating colorectal cancer initiation and progression. Semin Cell DevBiol 2020;98:63-70.

37. Hazwani MY, Sharaniza A, Suddin LS, et al. Metabolomics profiling on different stages of colorectal cancer: a systematic review. Malays J Med Sci 2018;25:16-34.

38. Mobasheri MB. Metabolomics in cancer. Basic Clin Cancer Res 2019;11:82-83.

39. Miyanari N, Baba H. Cancer metabolism in gastrointestinal cancer. J Cancer Metastasis Treat 2015;1:172-82.

40. Qiu Y, Cai G, Zhou B, et al. A Distinct Metabolic Signature of Human Colorectal Cancer with Prognostic Potential. Clin Cancer Res 2014;20:2136-2146.

41. Zhang J, Guo S, Li J, et al. Effects of high-fat diet-induced adipokines and cytokines on colorectal cancer development. FEBS Open Bio 2019;9:2117-25.

42. Yusof HM, Abrahim S, Zurinah WAN, et al. Metabolites profile of colorectal cancer cells at different stages. Int J Appl Pharm 2019;11:21-5.

43. Hagland HR, Jolma W. Molecular pathways and cellular metabolism in colorectal cancer. Dig Surg 2013;30:12-25.

44. Gu J, Xiao Y, Shu D, et al. Metabolomics analysis in serum from patients with colorectal polyp and colorectal cancer by 1 H-NMR spectrometry. Dis Markers 2019:1-12.

45. Notarnicola M, Caruso MG, Tutino V, et al. Nutrition and lipidomic profile in colorectal cancers. Acta Biomed
2018;89:87-96.

46. Zhang H, Zhang A, Miao J, et al. Targeting regulation of tryptophan metabolism for colorectal cancer therapy: a systematic review. RSC ADV 2019;9:3072-80.

47. Stewart HL. Geographic pathology of cancer of the colon and rectum. Cancer 1971;28:25-8.

48. Świderska M, Choromańska B, Dąbrowska E, et al. The diagnostics of colorectal cancer. ContempOncol 2014;18:1-6.

49. Quentmeier A, Moller P, Schwarz V, et al. Carcinoembryonic antigen, CA 19.9, and CA 125 in normal and carcinomatous human colorectal tissue. Cancer 1987;60:2261-6.

50. Koness RJ. CEA: is it of value in colorectal cancer? RI Med 1995;78:164-6.

51. Reaven GM. Banting lecture 1988. Role of insulin resistance in human disease. Diabetes 1988;37:1595-607

52. Laaksonen DE, Lakka HM, Niskanen LK, et al. Metabolic syndrome and development of diabetes mellitus: application and validation of recently suggested definitions of the metabolic syndrome in a prospective cohort study. Am J Epidemiol 2002;156:1070-77.

53. Furberg AS, Veierød MB, Wilsgaard T, et al. Serum high-density lipoprotein cholesterol, metabolic profile, and breast cancer risk. J Natl Cancer Inst 2004;96:1152-60.

54. Michaud DS, Liu S, Giovannucci E, et al. Dietary sugar, glycemic load, and pancreatic cancer risk in a prospective study. J Natl Cancer Inst 2002;94:1293-300.

55. Park JS, Choi GS, Jang YS, et al. Influence of obesity on the serum carcinoembryonic antigen value in patients with colorectal cancer. Cancer Epidemiol Biomarkers Prev 2010;19:2461-8

56. Herbeth B, Bagrel A. A study of factors influencing plasma CEA levels in an unselected population. OncodevBiol Med 1980;1:191-8.

57. Go VL.Carcinoembryonic antigen: clinical application. Cancer 1976;37:562-566

58. StockleyRA, Shaw J, Whitfield AG, et al. Effect of cigarette smoking, pulmonary inflammation, and lung disease on concentrations of carcinoembryonic antigen in serum and secretions. Thorax 1986;41:17-24.

59. Chevinsky AH. CEA in tumors of other than colorectal origin. SeminSurgOncol1991;7:162-6.

60. Bormer OP. Standardization, specificity, and diagnostic sensitivity of four immunoassays for carcinoembryonic antigen. ClinChem1991;37:231-6.

61. Fletcher RH.Carcinoembryonic antigen. Ann Intern Med 1986;104:66-73.

62. Reiter W, Stieber P, Reuter C, et al. Multivariate analysis of the prognostic value of CEA and CA19-9 serum levels in colorectall cancer. Anti Cancer Res 2000;20:5195-8.

63. Sato T, NishimuraG, NonomuraA, et al. Serological studies on CEA, CA 19-9, STn and SLX in colorectal cancer. HepatoGastroenterol1999;46:914-19.

64. Forones NM, M. Tanaka, CEA and CA 19-9 as prognostic indexes in colorectal cancer, Hepato-Gastroenterology, vol. 46, no. 26, pp. 905-908, 1999.

65. Liska V, HolubecLJr., TreskaV,et al. Dynamics of serum levels of tumour markers and prognosis of recurrence and survival after liver surgery for colorectal liver metastases, Anticancer Res2007;27:2861-4.

66. Webb P, Scott-Mackie L, Cunningham D, et al. The prognostic value of CEA, $\beta$ HCG, AFP, CA125, CA19-9 and c-erb b-2, $\beta \mathrm{HCG}$ immunohistochemistry in advanced colorectal cancer. AnnOncol1995;6:581-7. 
67. Morita S, NomuraT, FukushimaY, et al. Does serum CA19-9 play a practical role in the management of patients with colorectal cancer? Dis Colon Rectum2004;47:227-32.

68. Gasser M, GerstlauerC, Grimm M,et al. Comparative analysis of predictive biomarkers for therapeuticalstrategies in colorectal cancer. AnnSurgical Oncol2007;14:1272-84.

69. Kawamura YJ, Tokumitsu A, Sasaki J, et al. Colorectal Carcinoma with Extremely Low CA19-9. Gastroenterol ResPract2009:780263.

70. Lengauer C, Kinzler KW,Vogelstein B. Genetic instabilities in human cancers. Nature1998;396:643-9.

71. Grady WM, Pritchard CC. Molecular alterations and biomarkers in colorectal cancer. ToxicolPathol 2014;42:124-39.

72. Mäkelä JT, Laitinen ST,Kairaluoma MI. Five-year follow-up after radical surgery for colorectal cancer. Arch Surg 1995;130:1062-7.

73. Arveux I, Boutron MC, Arveux P, et al. Colon cancer in the elderly: evidence for major improvements in health care and survival. British J Cancer1997;76:963-7.

74. Obrand DI, Gordon PH. Incidence and patterns of recurrence following curative resection for colorectal carcinoma. Dis Colon Rectum 1997;40:15-24.

75. Vogelstein B, Fearon ER, Hamilton SR, et al. Genetic alterations during colorectal-tumor development. N Engl J Med1988;319:525-32.

76. Jones AM, Douglas EJ, Halford SE, et al. Array-CGH analysis of microsatellite-stable, near-diploid bowel cancers and comparison with other types of colorectal carcinoma. Oncogene2005;24:118-29.

77. Cancer Genome Atlas Network, CGAN: Comprehensive molecular characterization of human colon and rectal cancer. Nature2012;487:330-7.

78. Ogino S, Nosho K, Irahara N, et al. Prognostic significance and molecular associations of 18q loss of heterozygosity: A cohort study of microsatellite stable colorectal cancers. J ClinOncol2009;27:4591-8.

79. Sheffer M, Bacolod MD, Zuk O, et al. Association of survival and disease progression with chromosomal instability: A genomic exploration of colorectal cancer. ProcNatlAcadSci USA2009; 106:7131-6.

80. Fearon ER, Cho KR, Nigro JM, et al. Identification of a chromosome $18 \mathrm{q}$ gene that is altered in colorectal cancers. Science 1990;247:49-56.

81. Thiagalingam S, Lengauer C, Leach FS, et al. Evaluation of candidate tumour suppressor genes on chromosome 18 in colorectal cancers. Nature Genet 1996;13:343-6.

82. Eppert K, Scherer SW, Ozcelik H, et al. MADR2 maps to 18q21 and encodes a TGF $\beta$-regulated MAD-related protein that is functionally mutated in colorectal carcinoma. Cell 1996;86:543-52.

83. Zauber P, Sabbath-solitare M, Marotta SP and Bishop T: Loss of heterozygosity for chromosome $18 \mathrm{q}$ and microsatellite instability are highly consistent across the region of the DCC and SMAD4 genes in colorectal carcinomas and adenomas. J Appl Res2008;8:14-23.

84. Chang SC, Lin JK, Lin TC, Liang WY. Loss of heterozygosity: An independent prognostic factor of colorectal cancer. World JGastroenterol2005;11:778-84.

85. Iacopetta B, Grieu F, Amanuel B. Microsatellite instability in colorectal cancer. Asia Pac J ClinOncol 2010;6:260-9.

86. Woerner SM, Kloor M, von KnebelDoeberitz M, Gebert JF. Microsatellite instability in the development of DNA mismatch repair deficient tumors. Cancer Biomark 2006;2:69-86.

87. Strimpakos A, Syrigos K, Saif M. Pharmacogenetics and biomarkers in colorectal cancer. Pharmacogenomics J 2009;9:147-60.

88. Hoeijmakers JH. Genome maintenance mechanisms for preventing cancer. Nature 2001;411:366-74.

89. Thibodeau SN, French AJ, Cunningham JM, Tester D, Burgart LJ, Roche PC, McDonnell SK, Schaid DJ, Vockley CW, Michels VV, Farr GH Jr, O'Connell MJ. Microsatellite instability in colorectal cancer: different mutator phenotypes and the principal involvement of hMLH1. Cancer Res. 1998 Apr 15;58(8):1713-8. PMID: 9563488.

90. Ilyas M, Straub J, Tomlinson I, Bodmer WF. Genetic pathways in colorectal and other cancers. Eur J Cancer 1999; 35:19862002.

91. Trautmann K, Terdiman JP, French AJ, et al. Chromosomal instability in microsatellite-unstable and stable colon cancer. Clin Cancer Res 2006;12:6379-85.

92. Lynch PM. Current approaches in familial colorectal cancer: A clinical perspective. J NatlComprCancNetw 2006;4:421-30.

93. Weinberg RA: The biology of cancer. Baltimore, MD, Garland Science, 2006.

94. Iacopetta B, Li WQ, Grieu F, et al. BRAF mutation and gene methylation frequencies of colorectal tumours with microsatellite instability increase markedly with patient age. Gut 2006;55:1213-4. 